\title{
Superconductivity and Ferromagnetism
}

\section{in $\mathrm{La}_{0.67} \mathrm{Sr}_{0.33} \mathrm{MnO}_{3} / \mathrm{YBa}_{2} \mathrm{Cu}_{3} \mathrm{O}_{7}$ Nanosized Heterostructures}

\author{
H. Lochmajer ${ }^{a, b}$, M. Matusiak ${ }^{a, b}$, G. URbanik ${ }^{b, c}$, P. PRZyslupski ${ }^{d}$ And K. Rogacki ${ }^{a, b, c, *}$ \\ ${ }^{a}$ Institute of Low Temperature and Structure Research, PAS, Okólna 2, 50-422 Wrocław, Poland \\ ${ }^{b}$ Centre for Advanced Materials and Smart Structures, PAS, Okólna 2, 50-422 Wrocław, Poland \\ ${ }^{c}$ International Laboratory of High Magnetic Fields and Low Temperatures, PAS, \\ Gajowicka 95, 53-421 Wrocław, Poland \\ ${ }^{d}$ Institute of Physics, Polish Academy of Sciences, al. Lotników 32/46, 02-668 Warszawa, Poland
}

In this work we present recent experimental studies of heterostructures composed of ferromagnetic $\mathrm{La}_{0.67} \mathrm{Sr}_{0.33} \mathrm{MnO}_{3}$ (LSMO) and superconducting $\mathrm{YBa}_{2} \mathrm{Cu}_{3} \mathrm{O}_{7}$ (YBCO) multilayers grown by dc sputtering method. The transport and magnetic properties of the large set of heterostructures were investigated in a wide temperature range from 2 to $400 \mathrm{~K}$ and in fields up to $12.5 \mathrm{~T}$. The resistivity and the upper critical field were measured for samples with the plane active dimensions $1.5 \times 5 \mathrm{~mm}^{2}$. The Nernst effect was studied in the mixed state revealing the characteristic temperature dependence of the Nernst signal which was correlated with variation of the upper critical field. We have observed an influence of the magnetic LSMO layer on the superconducting properties of the adjacent YBCO layer which consists in a substantial reduction of the superconducting critical temperature and a small lowering of the upper critical field.

DOI: 10.12693/APhysPolA.130.588

PACS/topics: 74.25.fc, 74.25.fg, 74.78.Fk

\section{Introduction}

Nanosized ferromagnet/superconductor (FM/SC) heterostructures are an attractive model system for studying unusual superconducting properties and the interaction between magnetism and superconductivity in lowdimensional structures [1-3]. In these materials interspersed two-, three-, and multilayers of a strictly controlled thickness and the desired $\mathrm{FM} / \mathrm{SC}$ configuration can interact in different ways and with tuned strength. This allows one to carry out advanced research and verify various scenarios. Due to the complexity of the considered phenomena, occurring in the strongly correlated electron systems, and due to high anisotropy of the investigated materials, it is necessary to obtain layers with well-defined epitaxy, which can be achieved by modern methods of synthesis and with using oriented substrates. In this paper we present results of the research of FM/SC epitaxially grown heterostructures composed of manganites and cuprates with ferromagnetic $\left(T_{\mathrm{C}} \approx 350 \mathrm{~K}\right)$ and superconducting $\left(T_{c} \approx 90 \mathrm{~K}\right)$ ordering temperatures. Thus, the interaction between magnetism and superconductivity could be studied also at temperatures of liquid nitrogen, which is of practical importance.

\section{Experimental}

Samples of $\quad \mathrm{La}_{0.67} \mathrm{Sr}_{0.33} \mathrm{MnO}_{3} / \mathrm{YBa}_{2} \mathrm{Cu}_{3} \mathrm{O}_{7}$ thin films have been deposited epitaxially on (100) $\left[\left(\mathrm{LaAlO}_{3}\right)_{0.3}\left(\mathrm{Sr}_{2} \mathrm{TaAlO}_{6}\right)_{0.7}\right]$ (LSAT) substrates $\left(0.5 \times 5 \times 20 \mathrm{~mm}^{3}\right)$ by dc sputtering described

\footnotetext{
*corresponding author; e-mail: k.rogacki@int.pan.wroc.pl
}

elsewhere [4]. Samples with the thickness of the ferromagnetic $\mathrm{La}_{0.67} \mathrm{Sr}_{0.33} \mathrm{MnO}_{3}$ (LSMO) layer varied from $12 \mathrm{~nm}$ (L12) to $280 \mathrm{~nm}$ (L280) and the superconducting $\mathrm{YBa}_{2} \mathrm{Cu}_{3} \mathrm{O}_{7}$ (YBCO) layer varied from $20 \mathrm{~nm}$ (Y20) to $450 \mathrm{~nm}$ (Y450) were obtained. The number of layers was changed from 1 to 3 achieving following combinations: LSAT/LSMO, LSAT/YBCO, LSAT/LSMO/YBCO, and LSAT/LSMO/YBCO/LSMO. To improve the matching of the lattice constants between the substrate and YBCO compound, YBCO "monolayers" were also obtained on $40 \mathrm{~nm}$ thick PrBCO layer, which is not superconducting. In this work the results for only several samples from the series have been presented. The magnetic and transport properties have been studied with the Quantum Design MPMS and PPMS platforms, and the Oxford Instruments Cryostat with the home-made insert. Both ac susceptibility and dc magnetization were used to characterize the nanometers thick samples, for the magnetic field oriented parallel and perpendicular to the $c$-axis of LSMO and YBCO. Resistivity was measured with the four-probe method by using the ac $(17-19 \mathrm{~Hz})$ and dc technique to obtain $R(T)$ and $H_{c 2}(T)$ dependences in fields up to $12.5 \mathrm{~T}$. The Nernst effect was also studied, for samples clamped between two phosphor bronze blocks with two thermometers and two resistive heaters attached to the blocks [5]. Scanning tunneling microscopy (STM) images have been taken to show the topography of the investigated samples and prove their homogeneity. All presented data have been obtained for films in magnetic saturation, since magnetization measurements show that the ferromagnetic layer saturates in fields above $0.5 \mathrm{~T}$. 


\section{Results and discussion}

STM studies were performed for several samples to show a degree of horizontal and vertical uniformity of the surface layer. Figure 1 shows the STM image and the linear profile of the L90Y50 bilayer revealing a steplike structure of the YBCO layer with a maximum change of the thickness less than $4 \mathrm{~nm}$. Average height of the observed steps is about $1.2 \mathrm{~nm}$, which is the $c$-axis unit cell constant of the YBCO compound. This picture is typical for larger crystallites, independently of which part of the sample $\left(5 \times 10 \mathrm{~mm}^{2}\right)$ is studied. Uniformity of smaller crystallites is better. Heterostructures of the similar type show similar properties. Only high quality samples, characterized by the corresponding $R(T)$ dependence and sharp transition to the superconducting state (YBCO layer) have been taken for further investigations.
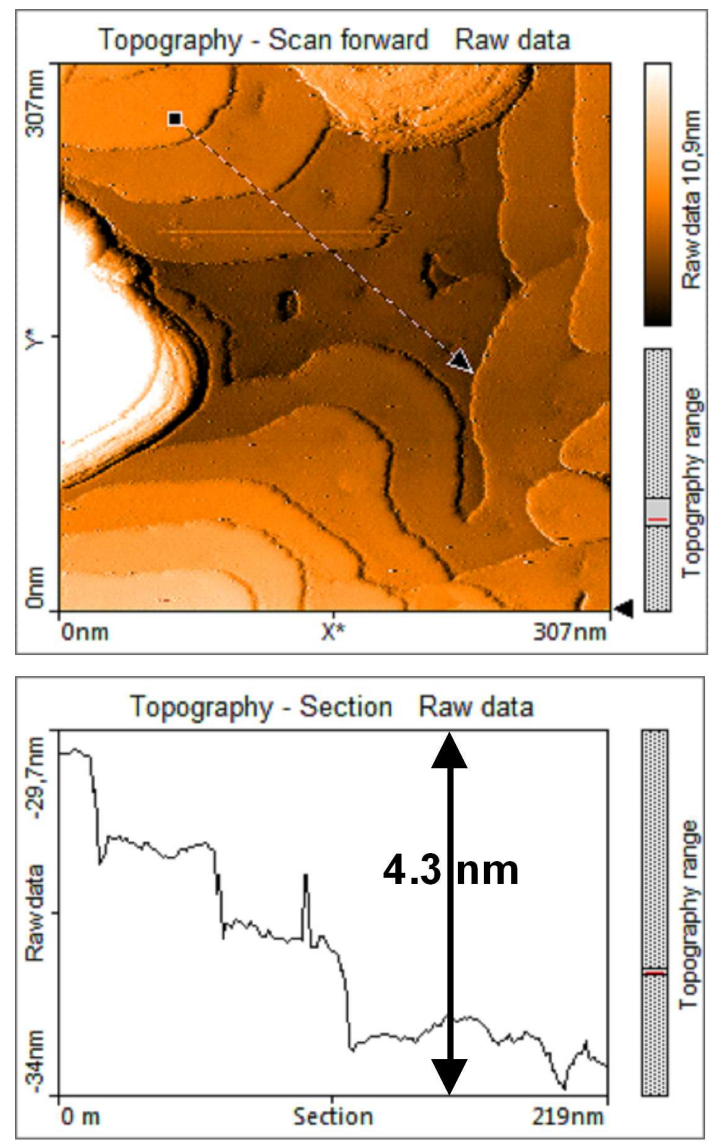

Fig. 1. STM image of the L90Y50 bilayer showing a step-like structure of the surface YBCO layer; the scanned area is $307 \times 307 \mathrm{~nm}^{2}$. Bottom part shows the STM linear profile taken along the dashed arrow. Average height of the observed three steps is about $1.2 \mathrm{~nm}$, which is the $c$-axis unit cell constant of the YBCO compound.

Magnetization loops $M(H)$ for the L90Y50 bilayer are presented in Fig. 2, for field oriented parallel to the $c$ axis of the crystallographic structure. The loops have been taken at several constant temperatures from 5 to $200 \mathrm{~K}$. Below $90 \mathrm{~K}$, the $M(H)$ loops reveal supercon-

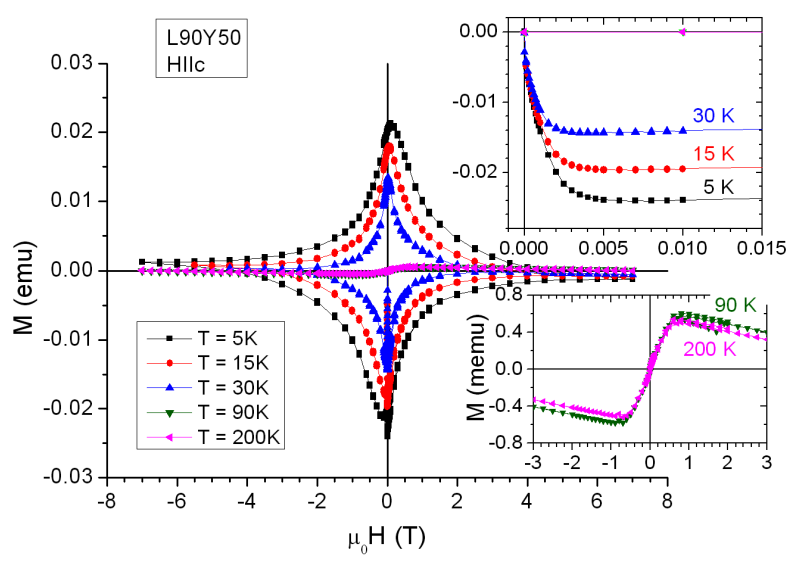

Fig. 2. Magnetization loops at several temperatures for the L90/Y50 bilayer with 90 and $50 \mathrm{~nm}$ thick FM and SC layer, respectively. The upper inset shows the virgin curves in the low field region from 0 to $15 \mathrm{mT}$. The lower inset presents reversible ferromagnetic curves in $\pm 3 \mathrm{~T}$ fields. For negative slope of the $M(H)$ curves (diamagnetism) observed in the state of saturation, a linear $M(H)$ signal from the sample holder is responsible. Measurements have been done for field oriented parallel to the $c$-axis of the LSMO and YBCO compounds.

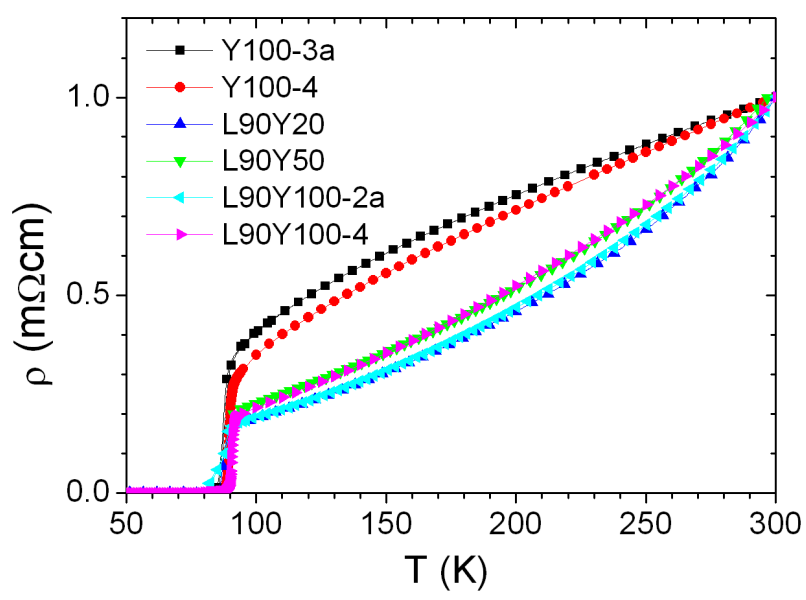

Fig. 3. Resistivity versus temperature for two SC monolayers (Y100-3a and Y100-4) and four FM/SC bilayers with a thickness of the FM layer fixed at $90 \mathrm{~nm}$ and a thickness of the SC layer varied from 20 through 50 to $100 \mathrm{~nm}$.

ducting properties which appear on the background of the ferromagnetic reversible curves. The loops decrease with increase of temperature and $M(H)$ becomes fully reversible at $T_{c} \approx 90 \mathrm{~K}$. Also the lower critical field, $H_{c 1}$, decreases with temperature, as it can be derived from the behavior of the virgin curves shown in the inset.

Resistivity $(\rho)$ versus temperature of the same L90Y50 bilayer and few other samples is presented in Fig. 3. The $\rho(T)$ dependences for bilayers (L90Y20, L90Y50, L90Y100-2a, L90Y100-4) show upward curvature, which means that the resistivity of $90 \mathrm{~nm}$ thick LSMO FM layer is lower than the resistivity of 20,50 , and $100 \mathrm{~nm}$ thick 


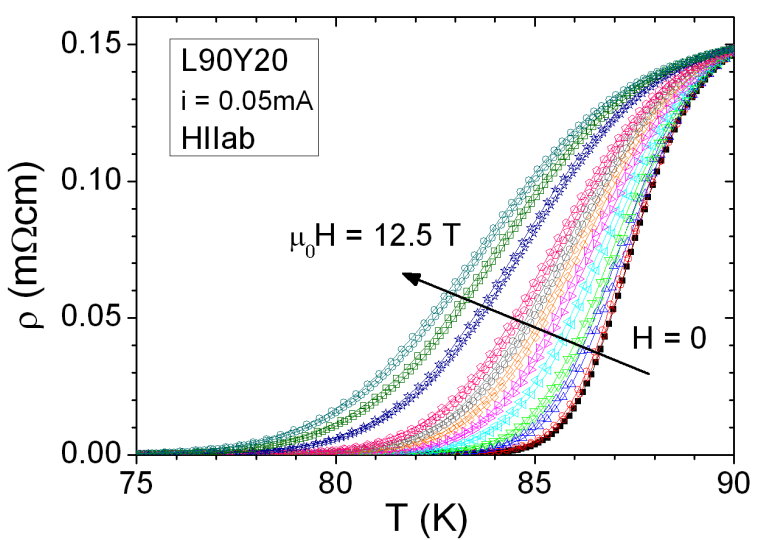

Fig. 4. Temperature dependence of the resistivity measured in magnetic fields from 0 to $12.5 \mathrm{~T}$, for the L90Y20 bilayer. Results have been obtained by sweeping the temperature up and down at constant magnetic field oriented parallel to the $a b$-plane of the bilayer. These and similar results were used to construct the phase diagram presented in Fig. 5, as described in the text.

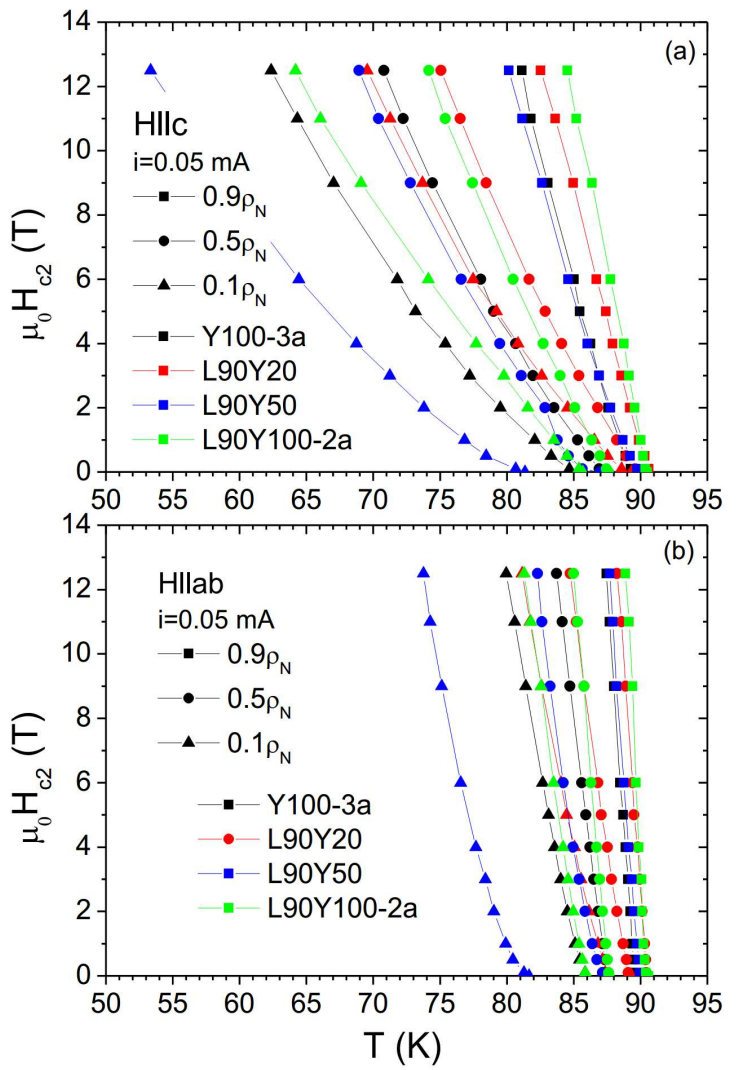

Fig. 5. Upper critical field (criterion $0.9 \rho_{N}$ ) and other characteristic fields (criterion $0.1 \rho_{N}$ and $0.5 \rho_{N}$ ) as a function of temperature for SC monolayer (Y100) and three FM/SC bilayers (L90Y20, L90Y50, L90Y100). Results have been obtained for field oriented (a) parallel and (b) perpendicular to the $c$-axis of the samples. Measuring current $(50 \mu \mathrm{A})$ was in $a b$-plane and perpendicular to the field.
YBCO SC layers and thus controls the conductivity for all measured bilayers. This is the reason why differences in the resistivity observed for the bilayers are small and may be even within the measurement error. Resistivity values and $\rho(T)$ dependences of two $100 \mathrm{~nm}$ thick YBCO monolayers (Y100-3a and Y100-4) are also very similar to each other and show differences within the measurement error. This result demonstrates good control of the synthesis conditions and proves high reproducibility of the samples preparation process. The values of $\rho$ and $\rho(T)$ dependences are like those observed for YBCO bulk material (downward curvature), which additionally confirms high quality of the $100 \mathrm{~nm}$ thick monolayer samples.

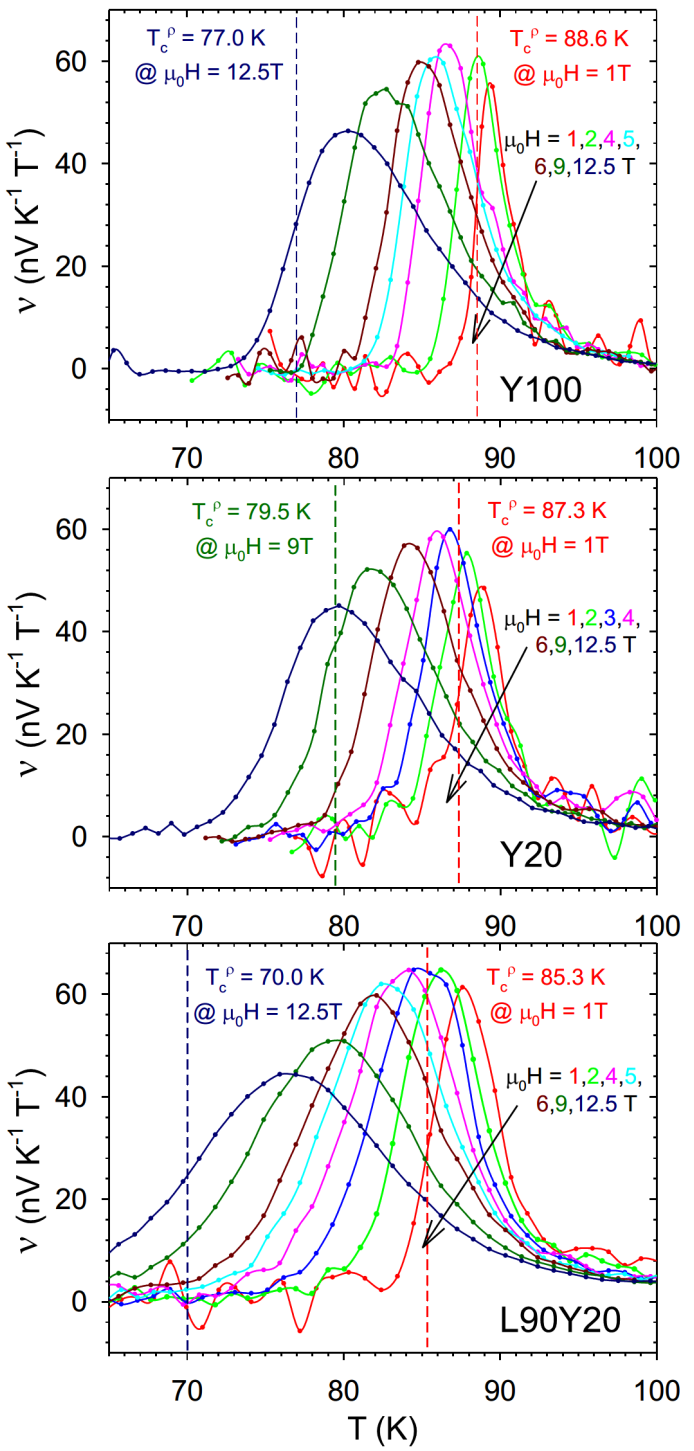

Fig. 6. Temperature dependences of the Nernst coefficient measured in various magnetic fields for three representative samples (Y100, Y20 and L90Y20). The dashed vertical lines mark the superconducting transition temperatures, taken at the inflection point of the resistance versus temperature curves obtained at given magnetic fields. 
Superconducting properties of the SC monolayers and FM/SC bilayers were characterized by the temperature dependence of the upper critical field obtained from the measurements of $\rho(T)$ in magnetic field. An example of the results is shown in Fig. 4, for the L90Y20 bilayer. Temperature dependences of some characteristic fields have been obtained by using different definitions: the specific temperatures were taken at $0.1,0.5$, and 0.9 of the normal state resistivity, $\rho_{N}$, for the $\rho(T)_{H}$ curves, where the criterion $0.1 \rho_{N}$ gives $H^{*}$, linked to the irreversibility field, and $0.9 \rho_{N}$ is believed to show the $H_{c 2}$ values. Figure 5 presents the upper critical field as a function of temperature for one SC monolayer and three $\mathrm{FM} / \mathrm{SC}$ bilayers. Results were obtained for the magnetic field oriented parallel and perpendicular to the $c$-axis of the samples, thus the anisotropy of $H_{c 2}$ can be estimated. This anisotropy is equal to about 6 for the Y100 monolayer and decreases to about 3 for the L90/Y100 bilayer. Figure 5 shows that $H_{c 2}$ for the $\mathrm{FM} / \mathrm{SC}$ bilayers reduces with decreasing thickness of the YBCO layer, which demonstrates the increased influence of the FM LSMO layer. No notable reduction of the $H_{c 2}$ values with decreasing thickness of the YBCO layer has been observed for high quality YBCO monolayers.

Temperature dependence of the Nernst coefficient, $\nu=$ $E / B \nabla T$, where $B$ is magnetic flux density and $E$ is transversal voltage, is shown in Fig. 6, for a few representative samples. More details about the Nernst effect in FM/SC bilayers can be found in Ref. [5]. For the $\nu(T)$ curves presented in Fig. 6 and analogous ones, the full width at half maximum has been determined to reveal the properties of the mixed state close to $T_{c}$. Main results are summarized in Fig. 7, which shows the $H_{c 2}(T)$ dependences and the width of the superconducting transition $\left(0.1 \rho_{N}\right.$ and $0.9 \rho_{N}$ criteria), together with the properties of the Nernst effect expressed as the temperature-field dependences of the maximum of the Nernst coefficient and the full width at half maximum obtained from the $\nu(T)_{H}$ curves. The results are shown for 20, 50, and $100 \mathrm{~nm}$ thick YBCO "monolayers" (two of them on $40 \mathrm{~nm}$ thick intermediate non-superconducting PrBCO layer) and for bilayers with similarly thick YBCO layers deposited on $90 \mathrm{~nm}$ thick LSMO layer.

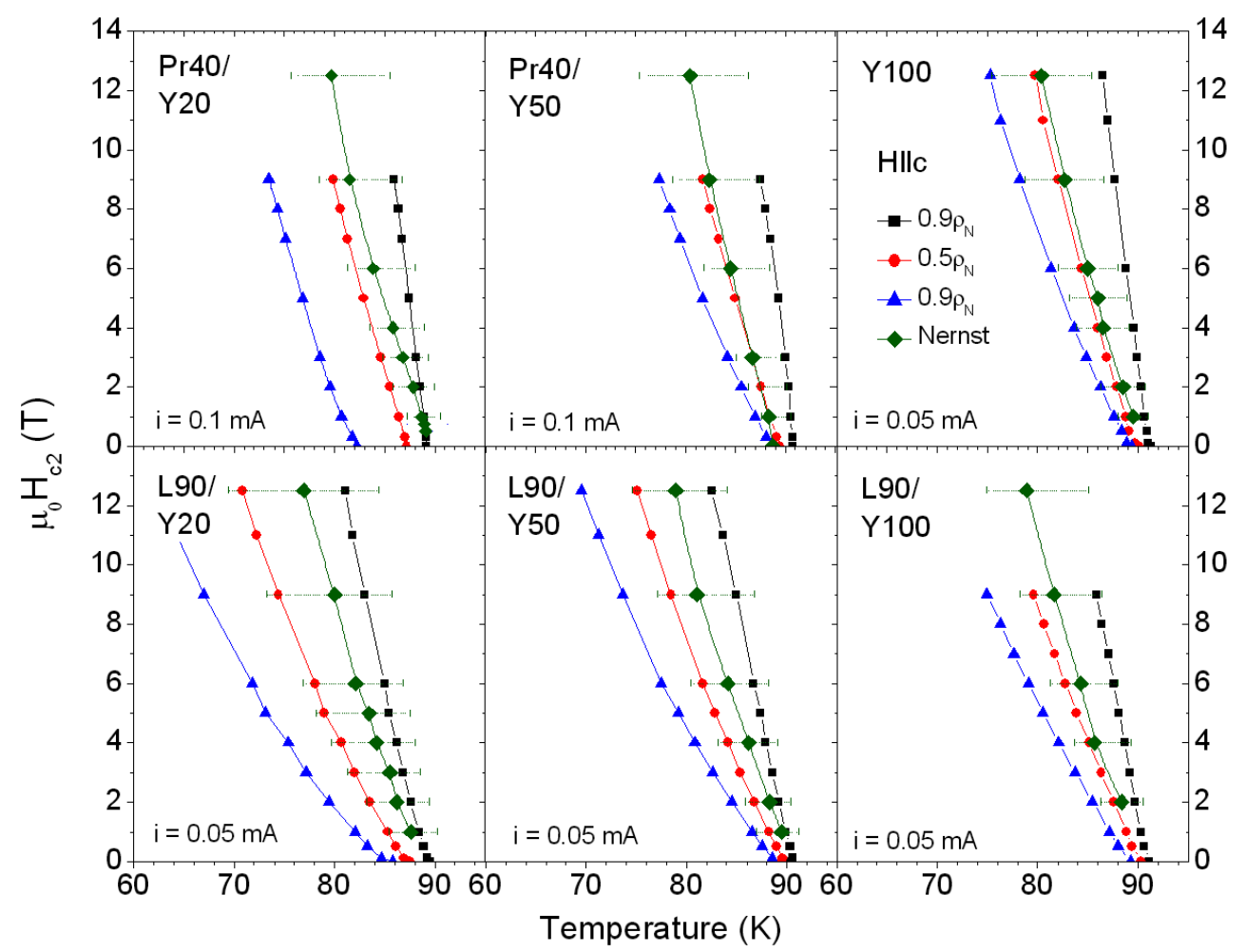

Fig. 7. Upper critical field (squares, $0.9 \rho_{N}$ criterion) and the width of the superconducting transition $\left(0.1 \rho_{N}\right.$ and $0.9 \rho_{N}$ criteria) as a function of temperature together with the Nernst effect properties shown as the temperature-field dependence of the maximum of the Nernst coefficient (diamonds) and the full width at half maximum (dashed horizontal lines) obtained from the $\nu(T)_{H}$ curves. The results are presented (upper part) for 20, 50, and $100 \mathrm{~nm}$ thick YBCO "monolayers" (two of them on $40 \mathrm{~nm}$ thick intermediate non superconducting Pr layer) and (lower part) for bilayers with similarly thick YBCO layers deposited on $90 \mathrm{~nm}$ thick FM LSMO layer.

Striking feature of the phase diagram shown in Fig. 7 is a clearly broader transition to the superconducting state, defined as a width $\Delta T_{c}$ (the criterion $0.1 \rho_{N}-0.9 \rho_{N}$ ), observed for $\mathrm{FM} / \mathrm{SC}$ bilayers, as compared with mono- 
layers. This is due to the additional magnetic field originating from a FM layer and resulting in the additional pair-breaking effect in the vicinity of the transition from superconducting to the normal state. Another significant effect, caused by the proximity of the FM layer, is an increase in the width of the transition, $\Delta T_{c}$, with decreasing thickness of the SC layer, which has been not observed for the monolayers. Nernst effect measurements show that the temperature dependence of the maximum and the full width at half maximum of the $\nu(T)_{H}$ curves is correlated to the $H_{c 2}(T)$ dependence. However, in the region of $H_{c 2}$, the Nernst signal is significantly different from zero. This result indicates the exceptional sensitivity of the Nernst effect to the appearance of superconducting vortices even without a long-range coherence. This proves the suitability of the Nernst effect to study the vortex properties close to and above the transition to the superconducting state.

\section{Conclusions}

Magnetic and transport properties of several SC monolayers (YBCO) and $\mathrm{FM} / \mathrm{SC}$ bilayers (LSMO/YBCO) have been studied in the vortex liquid state, in magnetic fields up to 12.5 T. Samples with YBCO layer (monoand bilayers) are superconducting with the zero resistance below the transition to the superconducting state. Both mono- and bilayers show the Meissner state and full shielding in low magnetic fields. The ferromagnetic LSMO layer increases the energy dissipation caused by movement of vortices in the YBCO layer, and has small detrimental effect on the upper critical field, $H_{c 2}$, and larger on the irreversibility field, $H_{\text {irr }}$. This results in the broadening of the flux-flow region and has been attributed to the influence of the large out-of-plane stray field in the domain walls, which can induce weakly pinned vortices in the YBCO layer and lead to additional energy dissipation $[6,7]$. The temperature dependence of the onset of the Nernst signal (at the normal state side) correlates with the temperature dependence of $\mathrm{H}_{c 2}$, and this signal turns to zero at temperatures much above the irreversibility field. Therefore, the Nernst effect can be undoubtedly used to study the vortex properties at temperatures close to the superconducting transition.

\section{Acknowledgments}

The work was supported by WRC EIT+ within the project NanoMat (P2IG.01.01.02-02-002/08) co-financed by the European Operational Programme Innovative Economy (1.1.2).

\section{References}

[1] C.L. Chien, D.H. Reich, J. Magn. Magn. Mater. 200, 83 (1999).

[2] J. Linder, J.W.A. Robinson, Nat. Phys. 11, 307 (2015).

[3] S.W. Huang, L.A. Wray, Horng-Tay Jeng, V.T. Tra, J.M. Lee, M.C. Langner, J.M. Chen, S. Roy, Y.H. Chu, R.W. Schoenlein, Y.-D. Chuang, J.-Y. Lin, Sci. Rep. 5, 16690 (2015).

[4] P. Przyslupski, I. Komissarov, W. Paszkowicz, P. Dluzewski, R. Minikayev, M. Sawicki, J. Appl. Phys. 95, 2906 (2004).

[5] M. Matusiak, H. Lochmajer, P. Przysłupski, K. Rogacki, Supercond. Sci. Technol. 28, 115002 (2015).

[6] T. Hu, H. Xiao, C. Visani, J. Santamaria, C.C. Almasan, New J. Phys. 13, 033040 (2011).

[7] C. Bell, S. Turşucu, J. Aarts, Phys. Rev. B $\mathbf{7 4}$, 214520 (2006). 